\title{
Alternately aligned 2D heterostructures enabled by $d$ - spacing accessible, highly periodic accordion-like graphene oxide frameworks
}

\author{
Yingbo Ruan ${ }^{2 \dagger}$, Zedong Zhao ${ }^{1 \dagger}$, Yuanhang $\mathrm{Ge}^{1}$, Bo $\mathrm{Hu}^{1}$, Mengxiong $\mathrm{Li}^{1}$, Lei Dong ${ }^{1}$, \\ Jiajia Zhang ${ }^{1^{*}}$ and Hongbin $\mathrm{Lu}^{1^{*}}$
}

\begin{abstract}
Two-dimensional (2D) heterostructures hold great promise in designing integrated materials, while the current synthesis strategies still confront challenges for multilayer heterostructure construction and scale-up production. Here we report a generalized host-guest strategy based on nonexfoliated layered graphene oxide (LGO) to construct graphene-based heterostructures that consist of multilayered, alternately aligned graphene and metal oxide nanosheets. The 2D-aligned GOs and open interlayer spaces make LGO an ideal platform to create periodic 2D host frameworks. Polyetheramine oligomers covalently bond the adjacent GOs. The extended chain conformation endows the resulting accordionlike GO frameworks with high structural stability, periodicity and enlarged interlayer space. Owing to the high affinity of the open and well-arranged 2D channels toward guest precursors, a variety of high-quality heterostructures can be synthesized. Furthermore, a variety of exfoliated, ultrathin metal oxide nanosheets can also be prepared by removing the graphene skeleton. The flexible interlayer chemistry presented in this study paves a way toward the synthesis of a large family of graphene-inorganic/organic $2 \mathrm{D}$ heterostructures.
\end{abstract}

Keywords: 2D heterostructures, alternately aligned, accordionlike graphene oxide frameworks, $d$-spacing, metal oxide nanosheets

\section{INTRODUCTION}

Two-dimensional (2D) heterostructures consisting of disparate nanosheets stacked alternatively via van der Waals interactions open up a new material design approach $[1,2]$ that has demonstrated great promise in a large range of fields such as energy storage [3] and cat- alysis [4]. A variety of nanosheets [5-7], such as graphene (graphene oxide (GO)) [8-16], MXene [17,18], dichalcogenide $[9,15,16,18,19]$ and oxide $[8,10,11,13]$ have exhibited the merits of heterostructures including enhanced charge storage capacity, transfer efficiency $[10-13,18,19]$, improved $\mathrm{CO}_{2}$ electroreduction [20] and water splitting performance [21]. The current synthesis strategy involves two catalogues, liquid-phase assembly and in-situ growth/ transformation under confined conditions. Liquid-phase assembly creates heterostructures primarily through flowdirected layer-by-layer stacking $[3,9]$ or charge-induced flocculation of pre-exfoliated nanosheets $[6,10,11,14,21]$. The key challenge lies in the realization of layer-by-layer or layer-wise alternate stacking of disparate nanosheets. For the flow-directed assembly, such stacking can be achieved via spin-coating, dipping coating or inkjet printing [9]. It not only requires the stable dispersion of atomically thin nanosheets in solution, but also needs continuously repeated operation to obtain a macroscopic multilayer heterostructure film. Flocculation-induced heterostructures primarily exploit the electrostatic interaction between different nanosheets to achieve alternate stacking. Since the majority of nanosheets are negatively charged, positive charge modification usually needs to be conducted prior to flocculation [14]. Furthermore, such interlayer stacking is not strictly ordered due to the stochastic nature of electrostatic interactions [6]. In contrast, confined growth or transformation offers a possibility to attain well-defined face-to-face heterophase stacking. A variety of exfoliated nanosheets have been employed to construct sandwich heterostructures through surfaceconfined growth or transformation [5,7]. However, the

\footnotetext{
${ }^{1}$ State Key Laboratory of Molecular Engineering of Polymers and Department of Macromolecular Science, Fudan University, Shanghai 200438, China

2 Xi'an Aerospace Composites Research Institute, Xi'an 710025, China

$\dagger$ These authors contributed equally to this work.

* Corresponding authors (emails: hongbinlu@fudan.edu.cn (Lu H); zhangjiajia@fudan.edu.cn (Zhang J))
} 
content of heterophase interfaces in sandwich heterostructures is typically $30 \%$ less than that of layer-wise, alternately stacked counterparts. To optimize the performance of heterostructures, it is desired to establish a feasible route to accomplish layer-wise alternate stacking of disparate nanosheets. In this regard, interlayer confined growth in non-exfoliated layered structures provides an alternative option [5,7]. Recently, 2D layered MXenes have shown the viability of this idea, in which porous carbon- and $\mathrm{MoS}_{2}$-based heterostructures were created via pre-intercalation of precursors $[12,18]$. However, due to the limited interlayer space, there still exists self-stacking of MXene sheets in the resulting heterostructures.

To achieve layer-wise alternate stacking, two prerequisites need to be fulfilled simultaneously. (1) The precursor to form nanosheets should have sufficient driving force to diffuse or intercalate into the interlayer space, for instance, through charge transfer or hydrogen bond interactions [22]. (2) Every interlayer space between the adjacent nanosheets should be accessible so that the precursors are able to fully occupy the whole interlayer space for forming nanosheets [23]. In this point, an open framework structure consisting of well-separated individual nanosheets is a necessary premise, and it would have sufficient, adjustable interlayer spaces that allow the access of different precursors.

GO frameworks (GOFs) is a promising option for constructing graphene-based layer-wise heterostructures. However, the current strategy to synthesize GOFs are primarily based on either solvothermal or $\pi$ - $\pi$-induced self-assembly of exfoliated (reduced) GO (rGO) nanosheets [24-31], which usually lead to disordered stacking and non-uniform interlayer spaces. To enhance the structural stability, a variety of linkers, such as diamine [24,26-29], boronic acid [25], thiourea [30,31] and porphyrin [32] have been used to immobilize the adjacent nanosheets. However, the reported GOFs usually show limited $d$-spacings (typically, $0.71-2.6 \mathrm{~nm}$ ), which restricts the diffusion of reaction precursors into the interlayer space and the formation of order-aligned heterostructures.

Here we demonstrate a strategy to construct alternately aligned, graphene-based heterostructures based on nonexfoliated layered GO (LGO) with open, adjustable interlayer space. By introducing difunctional polyetheramine (PEA) oligomer, LGO was transformed to the structure-stable, highly periodic, accordion-like GOF (AGOF) with $d$-spacing up to $8.9 \mathrm{~nm}$. Furthermore, the $d$-spacing of AGOF can be tuned through changing PEA concentration and the polarity of solvent. Since PEA has the extended chain conformations and chelating effect to metal ions, the precursors of metal oxides (MOs) can enter and uniformly distribute within the interlayer space of AGOF, and transform to the oxide nanosheets through rapid precipitation reactions and subsequent thermal treatment. Such feature renders AGOF suitable to prepare diverse heterostructures containing alternately aligned graphene and MO nanosheets. Furthermore, a variety of exfoliated, ultrathin MO nanosheets can also be prepared by replacing the graphene skeleton.

\section{EXPERIMENTAL SECTION}

\section{Syntheses}

Synthetic procedures of AGOF. A typical AGOF preparation process is as follows: $2.0 \mathrm{~g}$ of PEA-2k (molecular weight $2 \mathrm{~kg} \mathrm{~mol}^{-1}$, named as PEA-2k) was dissolved in $50 \mathrm{~mL}$ water, and $50 \mathrm{~mL}$ of LGO water suspension (LGO concentration: $5 \mathrm{mg} \mathrm{mL}^{-1}$ ) was added to the above PEA$2 \mathrm{k}$ aqueous solution. The solution was slowly shaken and mixed well. The resulting mixed solution was left for 5 days with intermittent slow shaking, ensuring that PEA$2 \mathrm{k}$ molecules can fully diffuse into the interlayer space of LGO. The mixture was heated in a $98^{\circ} \mathrm{C}$-oil bath for $24 \mathrm{~h}$. The product was filtered through a 200 -mesh sieve and repeatedly washed with water to remove unreacted PEA$2 k$ to obtain the wet AGOF. By freeze-drying, the dry AGOF can be obtained. A series of AGOFs can be prepared by tuning the concentration of PEA-2k or the molecular weights (PEA-0.6k and PEA-0.9k).

Synthetic procedures of graphene/MO (G/MO) 2D heterostructures. Taking the preparation of $\mathrm{G} / \mathrm{ZnFe}_{2} \mathrm{O}_{4}$ heterostructures as an example: $4.04 \mathrm{~g}$ of iron nitrate nonahydrate $(0.01 \mathrm{~mol})$ and $1.49 \mathrm{~g}$ zinc nitrate hexahydrate $(0.005 \mathrm{~mol})$ were dissolved in deionized water. Dry AGOF $(20 \mathrm{mg})$ was added to the above mixed solution $\left(10 \mathrm{~mL}\right.$, where the $\mathrm{Fe}^{3+}$ concentration was $1 \mathrm{~mol} \mathrm{~L}^{-1}$ and $\left.\mathrm{Fe}^{3+} / \mathrm{Zn}^{2+}=2\right)$ and soaked overnight. After the AGOF particles had completely precipitated, they were filtered to obtain wet AGOF particles containing a large amount of $\mathrm{Fe}^{3+}$ and $\mathrm{Zn}^{2+}$. Subsequently, the AGOF particles were added to $100 \mathrm{~mL}$ of aqueous ammonia solution $\left(1 \mathrm{~mol} \mathrm{~L}^{-1}\right)$ under rapid stirring for $30 \mathrm{~min}$. The precipitate was collected and filtered through a 200-mesh sieve and repeatedly washed with deionized water. After freeze-drying, the dry AGOF/hydroxide composite was obtained. Finally, the composite was heated from room temperature to $600^{\circ} \mathrm{C}$ with a heating rate of $5^{\circ} \mathrm{C} \mathrm{min}^{-1}$ and held for $2 \mathrm{~h}$ under nitrogen and then cooled down to 
obtain the final $\mathrm{G} / \mathrm{ZnFe}_{2} \mathrm{O}_{4}$ composite. Keeping the concentration ratio of $\mathrm{Fe}^{3+}$ and $\mathrm{Zn}^{2+}$ in the mixed solution unchanged, the $\mathrm{G} / \mathrm{ZnFe}_{2} \mathrm{O}_{4}$ composites with different precursor concentrations were prepared. Following similar methods, $\mathrm{G} / \mathrm{TiO}_{2}, \mathrm{G} / \mathrm{MgO}, \mathrm{G} / \mathrm{Fe}_{3} \mathrm{O}_{4}$ and other $2 \mathrm{D}$ heterostructures can be synthesized. By similar method using freeze-drying, $\mathrm{G} / \mathrm{Co}_{3} \mathrm{O}_{4}, \mathrm{G} / \mathrm{Mn}_{2} \mathrm{O}_{3}$ and other $2 \mathrm{D}$ heterostructures can be synthesized.

Exfoliation of non-layered MO nanosheets: The asprepared G/MO nanocomposites were firstly annealed at a certain temperature in an air atmosphere to remove the graphene component to obtain layered MO nanosheet aggregates. The MO aggregates were then added to solvent such as water or alcohol and dispersed by water bath ultrasonic to exfoliate the non-layered MO nanosheets. The selection of the annealing temperature of each sample needs to consider the following two points: (1) the annealing temperature should be higher than the corresponding layered G/MO heterostructures, and the temperature of graphene complete decomposition can be determined by thermogravimetric analysis (TGA) test in air atmosphere, to ensure that graphite carbon content can be completely removed under the applied annealing condition; (2) the annealing temperature should not exceed the annealing temperature in the nitrogen atmosphere that is used to prepare G/MO heterostructures, in order to avoid the change of the crystal structure of the formed MOs in the air as much as possible. The annealing temperature of each sample mentioned in this study was as follows: $\mathrm{ZnFe}_{2} \mathrm{O}_{4}, \mathrm{TiO}_{2}$, and $\mathrm{MgO}: 600^{\circ} \mathrm{C} ; \mathrm{Fe}_{2} \mathrm{O}_{3}$ : $550^{\circ} \mathrm{C} ; \mathrm{Mn}_{2} \mathrm{O}_{3}: 450^{\circ} \mathrm{C}$; and $\mathrm{Co}_{3} \mathrm{O}_{4}: 400^{\circ} \mathrm{C}$. It should be noted that after the $\mathrm{G} / \mathrm{Fe}_{3} \mathrm{O}_{4}$ composite was annealed in air, the $\mathrm{Fe}_{2} \mathrm{O}_{3}$ nanosheets were finally obtained because of the $\mathrm{Fe}_{3} \mathrm{O}_{4}$ oxidation reaction.

\section{Instrumentation and characterization}

Field emission scanning electron microscopy (SEM) tests were performed with a Zeiss Ultra 55. Transmission electron microscopy (TEM) tests were performed using a transmission electron microscope of FEI company model Tecnai G2 20 TWIN. The solid sample was dispersed in ethanol, and sonicated in water bath for $30 \mathrm{~min}$ to enable a better dispersion. Then the dispersion was dropped on a copper mesh, and the solvent was evaporated at room temperature for further testing. High resolution TEM (HRTEM) and selected area electron diffraction (SAED) were tested using JEOL-2100 F field emission transmission electron microscope. The sample preparation was same as that of TEM. The optical microscopy $(\mathrm{OM})$ and polarized light microscopy (POM) images were tested with Leica DM 2500P optical microscope in normal mode and orthogonal polarization, respectively. A purple compensation film was added for testing in orthogonal polarization mode. X-ray diffraction (XRD) was performed using an X-ray diffractometer of PANalytical X'Pert PRO from PANalytical's company. A Cu Ka target $(\lambda=0.154 \mathrm{~nm})$ was used, and the scanning diffraction angle $2 \theta$ ranged from $2^{\circ}$ to $90^{\circ}$. TGA tests were conducted in TA PE Pyris-1 with a flow rate of $40 \mathrm{~mL} \mathrm{~min}^{-1}$ in nitrogen atmosphere with the heating rate of $10^{\circ} \mathrm{C} \mathrm{min}^{-1}$. For small-angle X-ray scattering (SAXS), $\mathrm{Cu}$ Ka target ( $\lambda$ $=0.154 \mathrm{~nm}$ ) was used, and the detector was $1.5 \mathrm{~m}$ away from the sample. All samples were tested at room temperature in transmission mode for $30 \mathrm{~min}$. The 1D SAXS curve was obtained by integrating the $2 \mathrm{D}$ scatter gram. Raman spectroscopy was tested with XPloRA laser Raman Spectrometer.

\section{RESULTS AND DISCUSSION}

\section{Synthesis and structure of AGOF}

Non-exfoliated LGO was used to prepare AGOF, the precursor of graphene-based heterostructures. LGO consists of well-aligned GO nanosheets that contain abundant oxygen functional groups and similar sheet size and nanometer-scale interlayer spacings [33]. The flexible interlayer chemistry makes LGO a unique precursor in designing $2 \mathrm{D}$ confined space. Inspired by the reticular chemistry [34], in which a well-defined periodic structure is usually composed of building blocks (such as GO units in LGO) and building bonds (covalent linker between two GO units), we propose a precisely adjusting method to control the 2D interlayer space of AGOF through crosslinking between the adjacent GO sheets. The typical synthetic process of AGOF is shown in Fig. 1a. Linear PEA-2k was used as a linker, because its amino end groups can react with the epoxy groups on GO through the ring-opening addition reaction. This enables the GO sheets in LGO to be immobilized to form structurally stable AGOF and avoids dissociation.

To illustrate the effectiveness of PEA for controlling the $d$-spacing of AGOF, OM and SAXS of pristine LGO and AGOF were used to observe their morphology and $d$ spacing differences. As shown in Fig. 1b, the waterswelled LGO reveals an accordion-like layered structure at macroscopic scale, but no scattering peaks were detected from SAXS (Fig. 1f), implying a wide distribution of $d$-spacing and poor periodicity. In contrast, the PEAlinked AGOF not only inherits the accordion-like structure (Fig. 1c-e) but also shows high periodicity (Fig. 1f). 

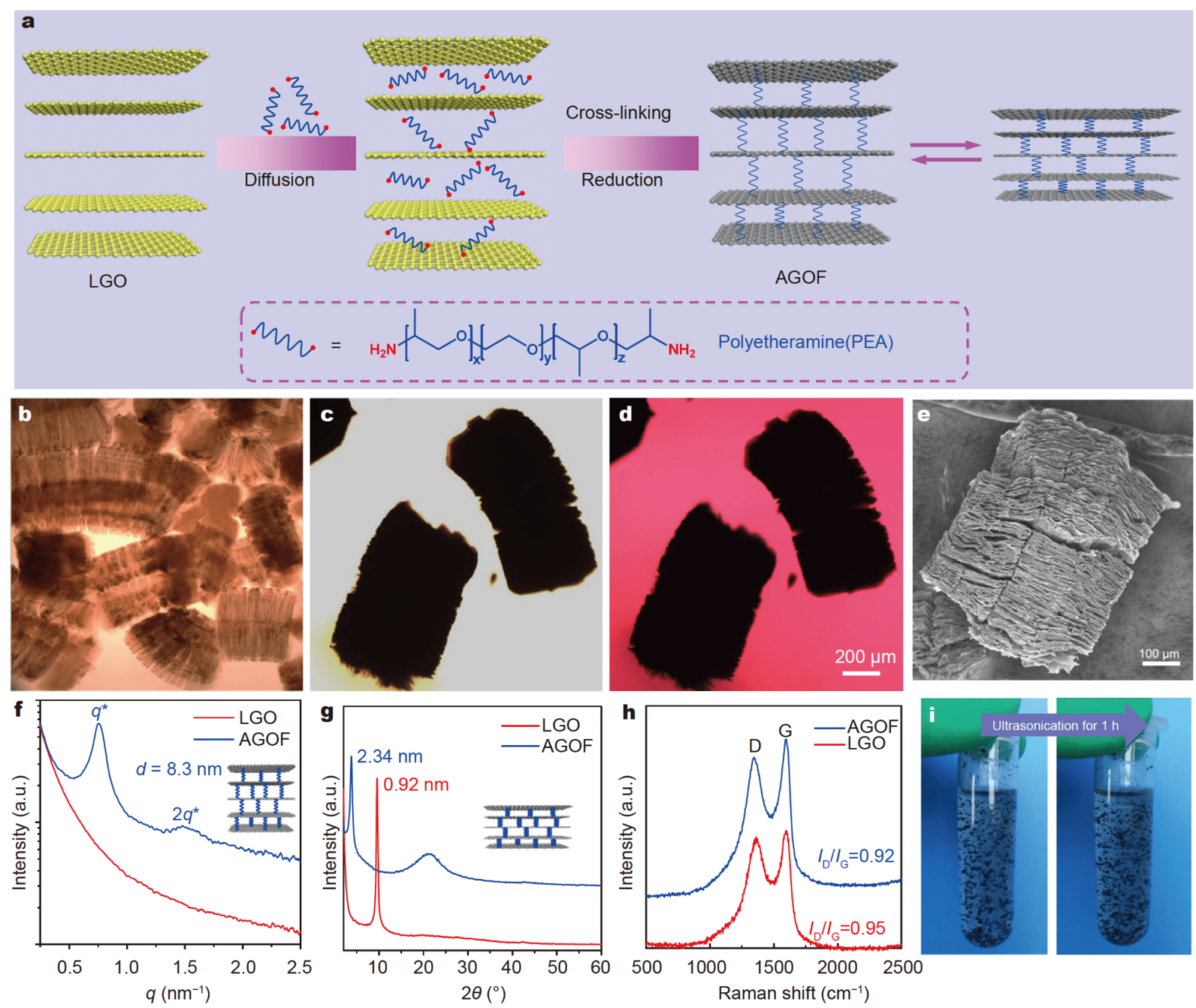

Figure 1 (a) Synthesis process of AGOF. (b) OM image of LGO. (c) OM and (d) polarized optical microscopy images of AGOF. (e) SEM image of dry-state AGOF particles. (f) 1D SAXS curves of wet-state LGO and AGOF samples. (g) XRD curves of wet-state LGO and AGOF samples. (h) Raman spectra of dry-state LGO and AGOF samples. (i) Comparison of AGOF suspension before and after $1 \mathrm{~h}$ ultrasonication.

A strong, narrow scattering peak appears at $q$ $=0.755 \mathrm{~nm}^{-1}$ (corresponding to the $d$-spacing of $8.3 \mathrm{~nm}$ ) for the wet-state sample, along with a second order scattering peak located at $q=1.5 \mathrm{~nm}^{-1}$. For the dried samples (Fig. 1g), the PEA-linked AGOF also possesses enlarged $d$-spacing of $2.34 \mathrm{~nm}$ compared with $0.92 \mathrm{~nm}$ of LGO. In addition, a diffraction peak at $21^{\circ}$, which arises from the stacking of $\mathrm{rGO}$, indicates that during grafting, PEA also induces the reduction of GO to some extent, consistent with the slightly decreased $I_{\mathrm{D}} / I_{\mathrm{G}}$ ratio (from 0.95 to 0.92 , Fig. $1 \mathrm{~h}$ ).

Further investigation with XPS and Fourier transform infrared (FTIR) on AGOF powders demonstrates that PEA chains are covalently bonded to GO sheets. The low- resolution XPS spectrum of the accordion-like AGOF shows the presence of $\mathrm{C}, \mathrm{O}$ and $\mathrm{N}$ species, with atomic contents of $69.7 \%, 28.9 \%$ and $1.4 \%$, respectively (Fig. S1a). Different from the single XPS peak at $398.7 \mathrm{eV}$ in $\mathrm{N}$ 1s spectrum of bulk PEA, we observed a new peak at $401.0 \mathrm{eV}$ from the $\mathrm{N}$ 1s spectrum of AGOF (Fig. S1b). This peak can be assigned to C-NH [35] formed via the nucleophilic substitution reaction between amine groups in PEA chains and three-membered epoxide rings in GO sheets [36]. Due to the difficulty in resolving $\mathrm{C}-\mathrm{O}$ and $\mathrm{C}-$ $\mathrm{N}$ from the XPS spectra of the $\mathrm{C} 1 \mathrm{~s}$ and the existence of a large amount of $\mathrm{C}-\mathrm{O}$ bonds in PEA chains, structural information driven from the $\mathrm{C} 1 \mathrm{~s}$ spectra is limited. The FTIR spectrum of AGOF exhibits the characteristic ab- 
sorption peaks of PEA (Fig. S1c), further proving the successful anchoring of PEA chains. The vibrational modes of oxygen-containing groups within the overlapped infrared frequency region of $800-1500 \mathrm{~cm}^{-1}$ have largely vanished after PEA crosslinking, owing to the ring-opening addition reaction between amino end groups and the epoxy groups on the GO sheets. In addition, AGOF still maintained its original structure even after sonication for $1 \mathrm{~h}$, further reflecting the existence of covalent bonds between PEA and GO sheets.

We next illustrated the difference between LGO and the exfoliated GO in constructing well-ordered interlayer spaces. Two kinds of frameworks were synthesized under the same reaction condition (Fig. S2a), based on LGO and the exfoliated GO sheets (denoted as GOF, distinguishing from AGOF), respectively. Owing to the high affinity of GO to PEA chains, the grafting amounts of PEA in GOF and AGOF show no significant difference (64.9 vs. $61.3 \mathrm{wt} \%$, Fig. S2g) and their chemical components are similar as well $\left(I_{\mathrm{D}} / I_{\mathrm{G}}: 0.93\right.$ vs. 0.92 , Fig. S2h). However, the resulting GOF reveals a disorder stacking structure (Fig. S2b-d) and no scattering peak was observed in the wet-state SAXS curves (Fig. S2e). After drying, the evaporation of water induced the partially ordered stacking of GO sheets, and therefore a diffraction peak corresponding to $d$-spacing of $2.86 \mathrm{~nm}$ was detected and a porous, disorder film formed (Fig. S2d, e). In contrast, the dried AGOF well retained an accordion-like particle morphology similar to the wet-state one (Fig. 1e), revealing a stronger diffraction peak at $2.34 \mathrm{~nm}$ (Fig. S2f) than GOF, suggesting the presence of more ordered stacking.

\section{Insight into the regulation mechanism of enlarged $d$ - spacing with high periodicity}

Constructing enlarged 2D $d$-spacing as well as high periodicity of AGOF are desirable to synthesize ordered alternately aligned heterostructures, as each separate open channel provides sufficient diffusion of reaction precursors. Apparently, the PEA linker is the key to control the $d$-spacing and periodicity. To give insight on the close connection between PEA and the resulting structure of AGOF, we firstly examined the effect of PEA concentration on the composition and structure of AGOF. Taking PEA-2k as an example, we studied the morphological evolution of AGOF with increasing PEA-2k concentration. As shown in Fig. S3, at the low concentrations of 1-2 $\mathrm{mmol} \mathrm{L}^{-1}$, the distortion and partial exfoliation of particles occurred and some were exfoliated, forming brown few-layer GO sheets. This arises from the fact that limited PEA molecules were unable to sufficiently immobilize the adjacent sheets, and thus part of them were dissociated. With the increase of PEA concentration, the structure integrity of AGOF particles was retained and less distortion and exfoliation occurred.

The effect of PEA concentration on the interlayer structure and periodicity of AGOF can be further elucidated by combining theoretical analysis and experimental observation. Fig. 2a shows a clear variation in the scattering signal of SAXS, where scattering intensity increases gradually with increasing PEA concentations $(C)$, but disappears at $C=50 \mathrm{mmol} \mathrm{L}^{-1}$. The similar trend also appears in the corresponding XRD results (Fig. 2b), implying that there is an optimum concentration for high periodicity of AGOF. Fig. 2c shows the correlation between the $d$-spacing and PEA concentration, where the interlayer spacings of both dry- and wet-state AGOFs gradually increase with increasing PEA concentrations. To further understand the role of PEA concentration on the resulting structure, the grafting density of PEA was then determined (Fig. 2d, refer to Fig. S4 and Table S1 for details). Combined with the above SXAS results (Fig. 2a), the grafting density governs the periodicity and interlayer spacing of AGOF to a large extent. According to the classic scaling theory [37], that is, $L \sim N b \sigma^{v}$, where $L$ is the thickness of grafting chains (approximately equals the interlayer spacing), $N$ is the number of Kuhn units, $b$ is the length of Kuhn units, $\sigma$ is the grafting density and $v$ is the power law exponent (refer to Supplementary information for details). As shown in Fig. 2e, in our case, the calculated $v=0.61(>>1 / 3)$ indicates that the PEA chains are in highly extended state within the interlayer [38]. In other words, increasing the graft density promoted the extension of PEA chains along the direction vertical to GO surfaces within the interlayer space [38], thus enlarging the interlayer space (Fig. 2c, d). Such chain vertical orientation and arrangement are also beneficial for building robust AGOF with high periodicity. Whereas at low PEA concentrations, the number of chains grafted on GO was limited so that PEA chains prefered to curl up randomly and distribute on GO sporadically [39], leading to reduced periodicity and interlayer spacing (Fig. 2a, c and d). At high PEA concentrations (above $50 \mathrm{mmol} \mathrm{L}^{-1}$ ), interchain entanglement may occur, resulting in limited long-range order and wide interlayer space distribution, evidenced by the significantly broadened scattering peaks upon increasing PEA concentrations. In addition, the molecular weight of PEA also reveals a critical influence on the periodicity of interlayer spacings. Owing to the non-exfoliated, interlayer separate nature of LGO in so- 

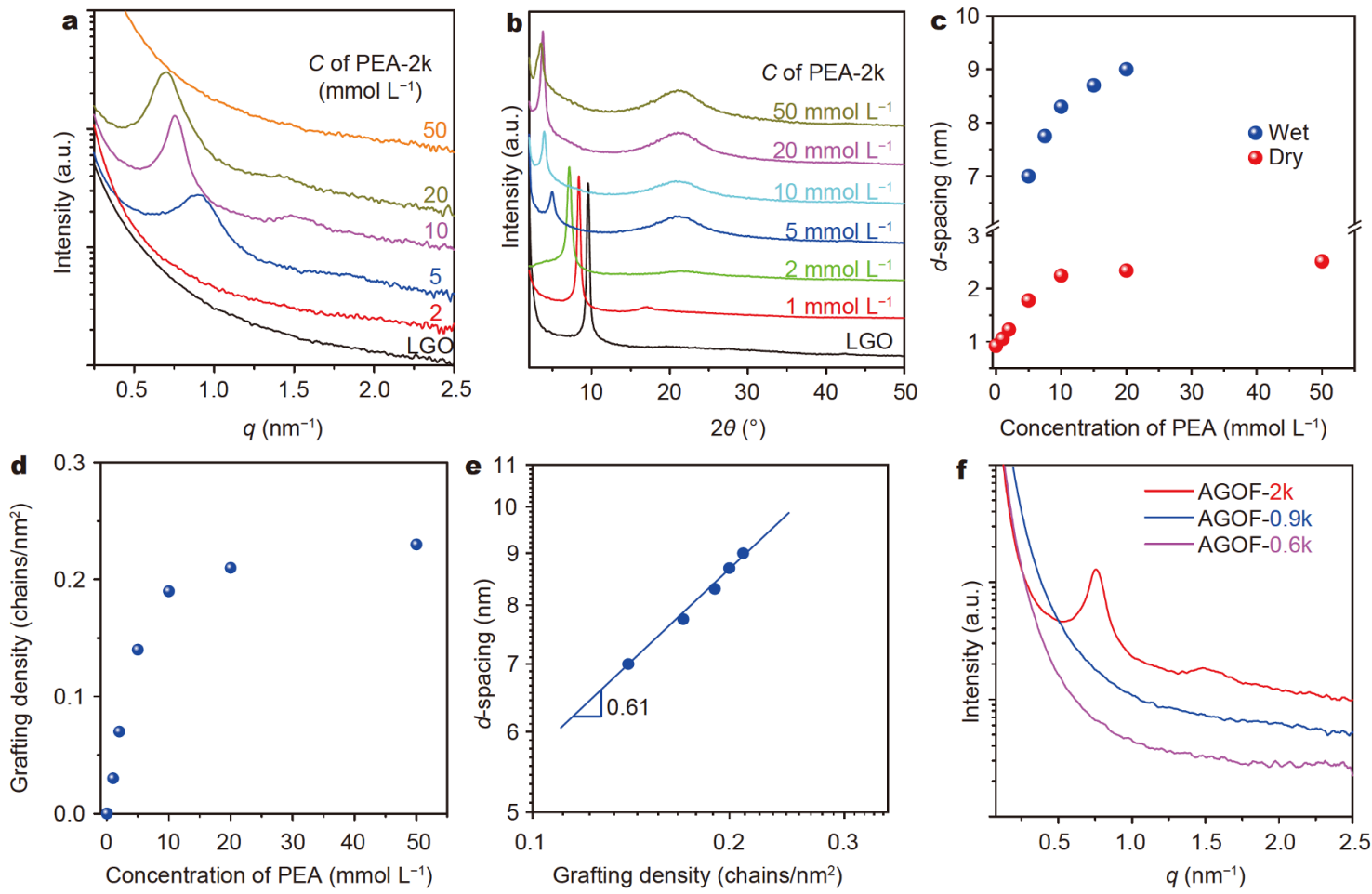

Figure 2 Effect of PEA concentration on the structure periodicity and interlayer spacing of AGOF. (a) SAXS and (b) XRD curves of LGO and AGOF samples prepared with different PEA concentrations. (c) The $d$-spacings of AGOF in wet and dry states as a function of PEA concentration. (d) Grafting density changes with PEA concentration. (e) $d$-spacing of the wet-state sample changes with grafting density. (f) SAXS curves of AGOF-2k, AGOF-0.9k and AGOF-0.6k. The PEA concentration is $10 \mathrm{mmol} \mathrm{L}^{-1}$.

lution, PEA with lower molecular weight possessed shorter chain length and thus cannot bridge two adjacent GO sheets, so that they were mostly grafted on GO surface in tail- or loop-grafting manners [24], resulting in poor periodicity (Fig. $2 \mathrm{f}$ and Fig. S5).

We next clarified the controlled mechanism of conformation-state of PEA chain on the periodicity and interlayer spacing via manipulating the content and type of solvents. Considering the dependence of chain conformation on solvent, we firstly examined the effect of water content on the $d$-spacing. As shown in Fig. S6, a highly reversible expansion/contraction process was observed with time of drying and re-wetting (Fig. S7c). SAXS results reveal that the $d$-spacing increases from 2.3 to $8.9 \mathrm{~nm}$ as the water content increases from 11.0 to 95.0 wt\% (Fig. 3a). This implies that the presence of water promotes the extension of PEA chains, which enables the $d$-spacing to be tuned in a wide range. If all water molecules enter the interlayer and form a $\mathrm{PEA} / \mathrm{H}_{2} \mathrm{O}$ mixture layer, the interlayer distance $d$ and water content $x$ could meet $d=(0.419 x+2.34) /(1-x)$ (Fig. 3b, refer to Fig. S8 for the details). However, it works only at $x<60 \%$, and when $x>60 \%$, a deviation appears between the calculated and experimental results, implying that extra water would have no contribution to the extension of PEA chains (Fig. S8). In addition, given that different solvents have different affinities to PEA chains, the $d$-spacing can also change when changing the polarity of solvent (Fig. $3 \mathrm{c}$ and Fig. S7). Since PEA chains are highly hydrophilic, the $d$ spacing in organic solvents gradually decreases with weakened polarity. The weakened SXAS signal can be explained by that partial PEA chains curl up and overlap which result in reduced periodicity. As shown in Fig. 3c, $\mathrm{d}$, besides water, $\mathrm{N}, \mathrm{N}$-dimethyl formamide can well dissolve PEA and thus AGOF exhibits $d$-spacing of $7.3 \mathrm{~nm}$, whereas in $n$-hexane it is just $2.7 \mathrm{~nm}$, reflecting contracted chain conformations.

\section{Synthesis of alternately aligned heterostructures}

AGOF provides a platform to synthesize diverse graphene-based heterostructures due to its highly accessible interlayer space. The extended chain conformation and hydrophilic nature of PEA allow a variety of metal ions to diffuse into the interlayer space and then transform to 

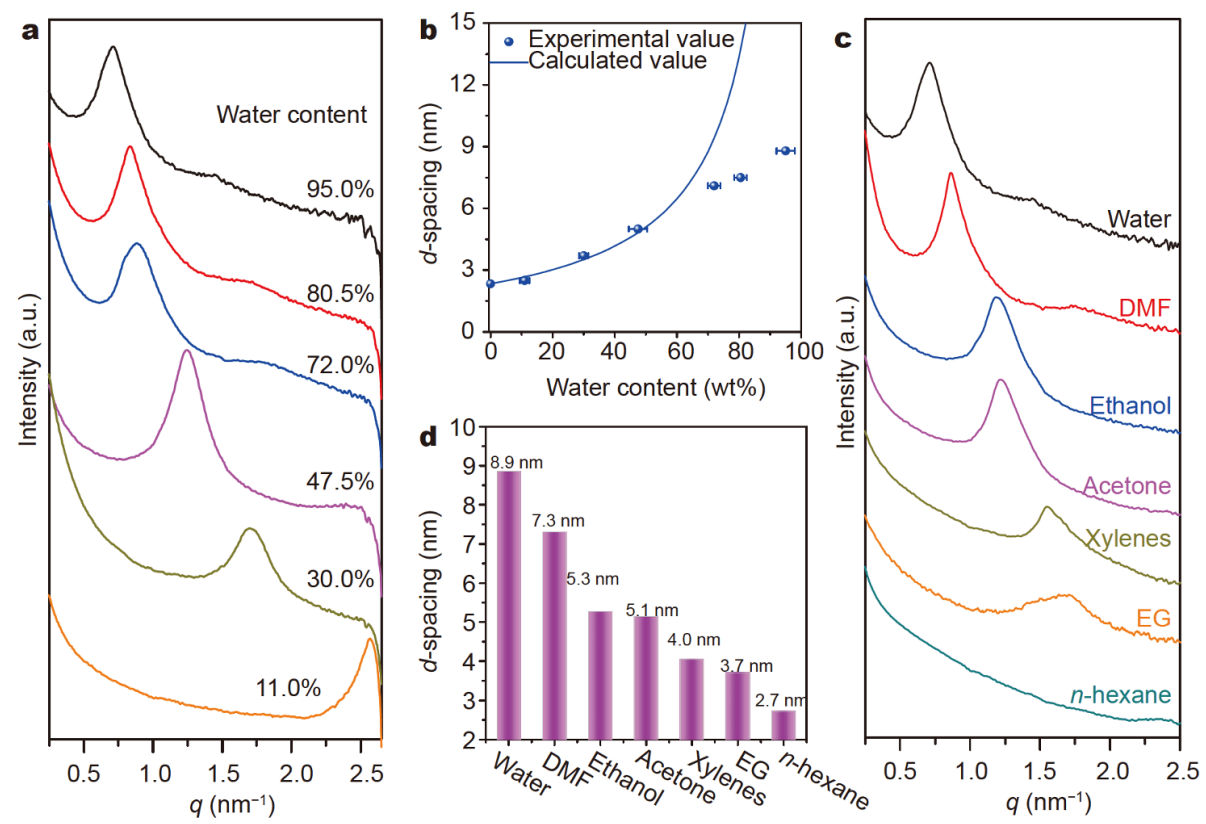

Figure 3 (a) 1D SAXS curves of AGOF-2k at different water contents. (b) Interlayer spacing of AGOF-2k changes with water content. (c) SAXS curves of dried AGOF-2k-20 after rewetting with different solvents. (d) Effect of different solvents on the interlayer $d$-spacing of AGOF-2k.

layered metal hydroxides or oxides. Employing AGOF and in-situ precipitation reaction, we synthesized several graphene-based, alternately aligned $\mathrm{MO}$ heterostructures, including $\mathrm{ZnFe}_{2} \mathrm{O}_{4}, \mathrm{TiO}_{2}, \mathrm{Fe}_{2} \mathrm{O}_{3}, \mathrm{Mn}_{2} \mathrm{O}_{3}$ and $\mathrm{Co}_{3} \mathrm{O}_{4}$. The corresponding process is illustrated in Fig. 4a. To control the precipitation reaction only to occur within the interlayer space, the salt solution outside AGOF was removed through filtration after the metal salt sufficiently diffused into the interlayer. The subsequent precipitation reactions and the chelating effect of ether bonds (of PEA) to metal ions enable the fast formation of layered metal hydroxides within AGOF.

We next gave an insight into the structure evolution of the graphene-based heterostructure during the synthetic process, by taking the study of the $\mathrm{AGOF} / \mathrm{ZnFe}_{2} \mathrm{O}_{4}$ heterostructure $\left(\mathrm{G} / \mathrm{ZnFe}_{2} \mathrm{O}_{4}\right)$ as an example. Fig. $4 \mathrm{~b}$ shows the SAXS curves of the intermediate products during the preparation process of $\mathrm{G} / \mathrm{ZnFe}_{2} \mathrm{O}_{4}$. The metal salt-containing AGOF (sample-W) has the $d$-spacing $(7.3 \mathrm{~nm}$ ) similar to that $(7.5 \mathrm{~nm})$ of AGOF. After transforming to the hydroxide-containing AGOF (sample-P), a wide scattering peak is observed despite the reduced intensity, indicating that it still maintains the periodic layered structure after chemical precipitation. The XRD patterns (Fig. 4c) of the dried samples confirm that the intermediate hydroxides $\mathrm{Fe}(\mathrm{OH})_{3}$ and $\mathrm{Zn}(\mathrm{OH})_{2}$ have been incorporated into each interlayer space of AGOF because there exist no characteristic diffraction peaks $\left(3.7^{\circ}\right.$ and $21.2^{\circ}$ ) of the dried AGOF (sample-D). The resulting composite after annealing at $600^{\circ} \mathrm{C}$ for $2 \mathrm{~h}$ shows the clear lattice structure consistent with $\mathrm{ZnFe}_{2} \mathrm{O}_{4}$ crystals (JCPDS 22-11012). Fig. $4 \mathrm{~d}$ reflects the effect of salt contents on the interlayer structure of AGOF. At low salt concentrations $\left(0.25\right.$ and $\left.0.5 \mathrm{~mol} \mathrm{~L}^{-1}\right)$, adjacent GO sheets were not completely separated so that a very weak diffraction peak at $21.2^{\circ}$ remained discernible, due to relatively low salt contents unable to fully occupy the open interlayer space. However, at higher salt contents $\left(1\right.$ and $\left.2 \mathrm{~mol} \mathrm{~L}^{-1}\right)$, $\mathrm{ZnFe}_{2} \mathrm{O}_{4}$ nanosheets well separate each GO layer in AGOF and no any diffraction peaks can be observed. This could be further supported by the TGA results, which show that the weight percent of $\mathrm{ZnFe}_{2} \mathrm{O}_{4}$ increases from $46.7 \%$ to $64.7 \%$ with increasing salt contents (Fig. $4 \mathrm{e}$ and Fig. S9).

The resulting $\mathrm{G} / \mathrm{ZnFe}_{2} \mathrm{O}_{4}$ heterostructure well retains the accordion-like framework morphology (Fig. 4f, g), exhibiting excellent structure stability. This primarily arises from the interlayer-bridged PEA chains, which also promotes the uniform distribution of the metal salt ions and the formation of regular, surface-smooth $\mathrm{ZnFe}_{2} \mathrm{O}_{4}$ nanosheets (Fig. 4h). Cross-section energy dispersive spectrometer analysis evidenced that $\mathrm{ZnFe}_{2} \mathrm{O}_{4}$ was confined within the interlayer space of graphene (Fig. S10). Due to high-temperature treatment (transformation from 


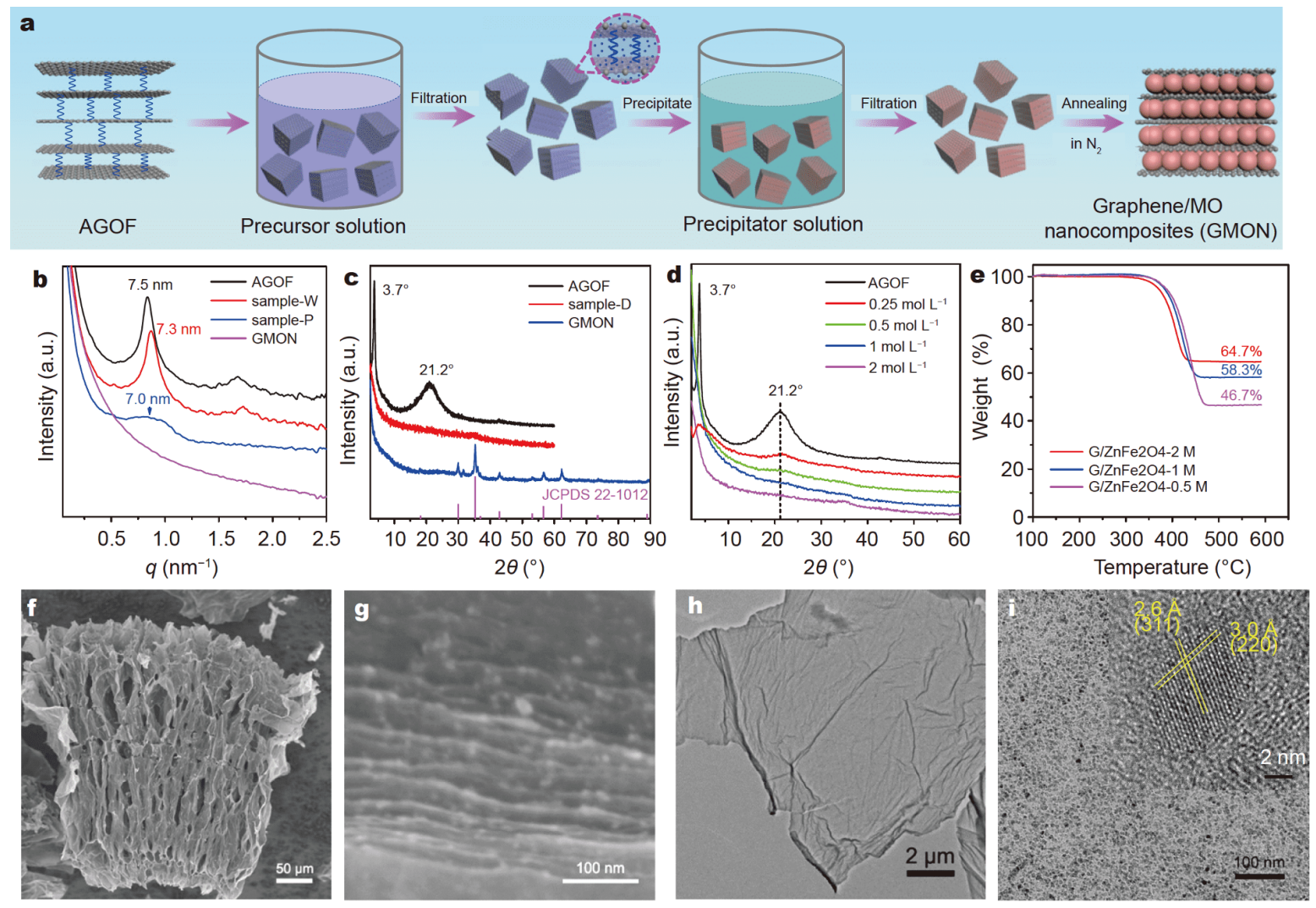

Figure 4 (a) Schematic for preparing alternately aligned graphene-based heterostructures by the rapid precipitation reaction. (b) SAXS and (c) XRD curves of samples under different synthetic processes. (d) XRD curves of sample-D prepared with different salt concentrations. (e) TGA curves of G/ $\mathrm{ZnFe}_{2} \mathrm{O}_{4}$ composites prepared in different salt concentrations, (f, g) SEM and (h, i) TEM images of G/ZnFe $\mathrm{O}_{4}$ heterostructure.

hydroxide to oxide), the resulting $\mathrm{ZnFe}_{2} \mathrm{O}_{4}$ nanosheets consist of nanocrystals $(\sim 10 \mathrm{~nm})$ distributed in graphene regions (Fig. 4i). From the HRTEM image, (311) and (220) facets of $\mathrm{ZnFe}_{2} \mathrm{O}_{4}$ are clearly identified, illustrating that the observed nanosheets are heterostructures other than graphene sheets. Their $2 \mathrm{D}$ characteristics can be further determined through removing graphene and liquid-phase exfoliation (Fig. 5a). Fig. 5b-d show a representative structure of $\mathrm{ZnFe}_{2} \mathrm{O}_{4}$ nanosheets after removing graphene, which still reveals an orderly-stacked framework morphology. Since there exist no PEAbridged graphene frameworks, they can be readily exfoliated in solution into individual nanosheets (Fig. 5e-h) with a thickness of $\sim 5 \mathrm{~nm}$ (Fig. 5i) and lateral size of several micrometers.

AGOF provides a new interlayer nanoreactor pathway for the fabrication of a wide range of graphene-based 2D heterostructures, which is different from those reported strategies based on surface- or spatial-confined templates [5-8]. Previously reported GOFs were generally synthesized through self-assembly of dispersed GO sheets that showed disordered stacking and non-uniform interlayer spaces [40-42]. Therefore, it is difficult to obtain alternately aligned 2D heterostructures with a long-range periodicity from these frameworks. Meanwhile, the swollen feature of AGOF when immersed in water allows for the effective intercalation and accumulation of reactive precursors due to the large interlayer distances, beneficial for constructing highly ordered heterostructures. In contrast, most GOFs reported so far show less swollen or non-swollen characteristics after being exposed into water, leading to limited $d$-spacings (typically, $0.71-2.6 \mathrm{~nm}$ ) and associated high energy barriers for ion diffusion, especially for those with large hydration radiuses. Non-exfoliated LGO largely simplifies the preparation procedure of GO, which can be obtained directly from graphite flakes and rapidly purified, without the need of pre-synthesis and assembly of GO sheets. In addition, the alternately aligned interlayer spaces enable AGOF to construct conveniently layer-wise aligned heterostructures, other than sandwich or randomly stacked structures. To demonstrate the structure advantage of 


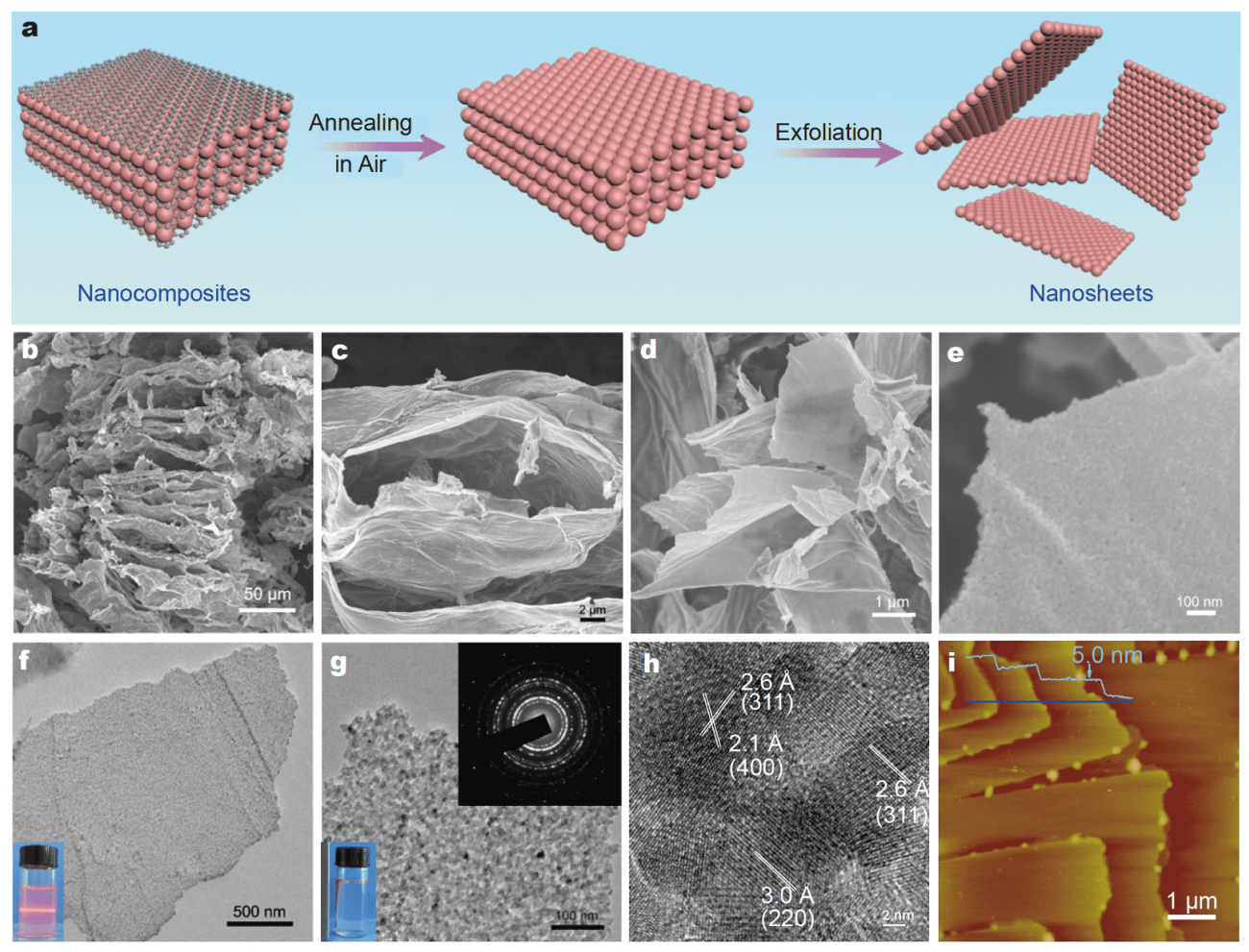

Figure 5 (a) Schematic for exfoliating MO nanosheets from G/MO heterostructures. (b, c) SEM images of layered $\mathrm{ZnFe}_{2} \mathrm{O}_{4}$ aggregates. (d, e) SEM images of exfoliated $\mathrm{ZnFe}_{2} \mathrm{O}_{4}$ nanosheets. (f, g) TEM, (h) HRTEM and (i) AFM image of $\mathrm{ZnFe}_{2} \mathrm{O}_{4}$ nanosheets. The inset of (f) shows the Tyndall effect of the $\mathrm{ZnFe}_{2} \mathrm{O}_{4}$ nanosheet dispersion, and the insets of $(\mathrm{g})$ are the magnetic test photo of $\mathrm{ZnFe}_{2} \mathrm{O}_{4}$ nanosheet dispersion and the corresponding SAED.

AGOF in constructing highly ordered 2D heterostructures, GO and GOF were further employed as nanoreactors for comparison (Fig. S11). The PEA linker enlarges the interlayer space into open $2 \mathrm{D}$ channels, facilitating the diffusion and uniform distribution of a variety of metal ions within the interlayer space, locking reaction precursors to form structure-stable $2 \mathrm{D}$ heterostructures. To demonstrate the generality, we also synthesized other nonlayered metal oxide heterostructures, including $\mathrm{TiO}_{2}, \mathrm{MgO}, \mathrm{Fe}_{2} \mathrm{O}_{3}, \mathrm{Mn}_{2} \mathrm{O}_{3}$ and $\mathrm{Co}_{3} \mathrm{O}_{4}$ (Figs S12-14). It is believed that such an industrially viable technical route contributes to the development of graphene-based 2D heterostructures in diverse fields. Meanwhile, such long-range-ordered heterostructures consist of alternating layers of MO sheets and graphene lamellae, which can potentially provide fast and smooth diffusion pathways for charge storage (Fig. S15).

\section{CONCLUSIONS}

We demonstrated a generalized method to construct layer-wise, alternately aligned graphene-based 2D heterostructures via the construction of highly periodic GOF with $2 \mathrm{D}$ open channels. Different from the existing strategies, which are mostly based on exfoliated GO, we employed non-exfoliated LGO with 2D-aligned GO to create alternately aligned interlayer nanoreactors. PEA was used to immobilize adjacent GO nanosheets to form structurally stable AGOF. This enables the $d$-spacing of AGOF to be enlarged via tuning the PEA content and the polarity of solvent while retaining high periodicity. Owing to the extended chain conformation of PEA and chelating capability to metal ions, the precursors of a variety of MOs can rapidly, uniformly distribute within the interlayer space of AGOF, forming alternately aligned graphene-based 2D heterostructures. Encouragingly, a series of ultrathin MO nanosheets can also be produced by removing the graphene skeleton. In view of highly accessible interlayer space and abundant functional groups of AGOF, a wide range of organic precursors with different functional substituents may also be used to synthesize other novel 2D heterostructures, including the emerging 2D materials such as metal-organic frameworks (MOFs), covalent organic frameworks (COFs). It is believed that flexible interlayer chemistry provides a practically viable route to synthesize a large family of graphene-inorganic/organic 2D heterostructures. The 
integrated advantages of AGOF make it a versatile material platform for both fundamental studies and device design.

\section{Received 4 August 2020; accepted 14 October 2020;} published online 5 January 2021

1 Geim AK, Grigorieva IV. Van der Waals heterostructures. Nature, 2013, 499: 419-425

2 Novoselov KS, Mishchenko A, Carvalho A, et al. 2D materials and van der Waals heterostructures. Science, 2016, 353: aac9439

3 Pomerantseva E, Gogotsi Y. Two-dimensional heterostructures for energy storage. Nat Energy, 2017, 2: 17089

4 Deng D, Novoselov KS, Fu Q, et al. Catalysis with two-dimensional materials and their heterostructures. Nat Nanotech, 2016, 11: 218230

5 Li Z, Zhang X, Cheng $\mathrm{H}$, et al. Confined synthesis of 2D nanostructured materials toward electrocatalysis. Adv Energy Mater, 2019, 10: 1900486

6 Xiong P, Sun B, Sakai N, et al. 2D superlattices for efficient energy storage and conversion. Adv Mater, 2020, 32: 1902654

7 Zhu Y, Peng W, Li Y, et al. Multiple roles of a heterointerface in two-dimensional van der Waals heterostructures: insights into energy-related applications. J Mater Chem A, 2019, 7: 2357723603

8 Zhu H, Xiao C, Cheng H, et al. Magnetocaloric effects in a freestanding and flexible graphene-based superlattice synthesized with a spatially confined reaction. Nat Commun, 2014, 5: 3960

9 McManus D, Vranic S, Withers F, et al. Water-based and biocompatible $2 \mathrm{D}$ crystal inks for all-inkjet-printed heterostructures. Nat Nanotech, 2017, 12: 343-350

10 Xiong P, Ma R, Sakai N, et al. Genuine unilamellar metal oxide nanosheets confined in a superlattice-like structure for superior energy storage. ACS Nano, 2018, 12: 1768-1777

11 Xiong $\mathrm{P}$, Zhang X, Zhang F, et al. Two-dimensional unilamellar cation-deficient metal oxide nanosheet superlattices for high-rate sodium ion energy storage. ACS Nano, 2018, 12: 12337-12346

12 Zeng C, Xie F, Yang X, et al. Ultrathin titanate nanosheets/graphene films derived from confined transformation for excellent $\mathrm{Na} / \mathrm{K}$ ion storage. Angew Chem Int Ed, 2018, 57: 8540-8544

13 Wang $\mathrm{X}, \mathrm{Li} \mathrm{Y}$, Wang $\mathrm{S}$, et al. $2 \mathrm{D}$ amorphous $\mathrm{V}_{2} \mathrm{O}_{5} /$ graphene heterostructures for high-safety aqueous $\mathrm{Zn}$-ion batteries with unprecedented capacity and ultrahigh rate capability. Adv Energy Mater, 2020, 10: 2000081

14 Cai X, Ozawa TC, Funatsu A, et al. Tuning the surface charge of 2D oxide nanosheets and the bulk-scale production of superlatticelike composites. J Am Chem Soc, 2015, 137: 2844-2847

15 Gao X, Qi J, Wan S, et al. Conductive molybdenum sulfide for efficient electrocatalytic hydrogen evolution. Small, 2018, 14: 1803361

16 Zhao C, Wang X, Kong J, et al. Self-assembly-induced alternately stacked single-layer $\mathrm{MoS}_{2}$ and N-doped graphene: A novel van der Waals heterostructure for lithium-ion batteries. ACS Appl Mater Interfaces, 2016, 8: 2372-2379

17 Wang J, Tang J, Ding B, et al. Hierarchical porous carbons with layer-by-layer motif architectures from confined soft-template selfassembly in layered materials. Nat Commun, 2017, 8: 15717

18 Chen $\mathrm{C}$, Xie X, Anasori B, et al. $\mathrm{MoS}_{2}$-on-MXene heterostructures as highly reversible anode materials for lithium-ion batteries.
Angew Chem Int Ed, 2018, 57: 1846-1850

19 Ma M, Zhang S, Yao Y, et al. Heterostructures of 2D molybdenum dichalcogenide on 2D nitrogen-doped carbon: superior potassiumion storage and insight into potassium storage mechanism. Adv Mater, 2020, 32: 2000958

20 Han P, Wang Z, Kuang M, et al. 2D assembly of confined space toward enhanced $\mathrm{CO}_{2}$ electroreduction. Adv Energy Mater, 2018, 8: 1801230

21 Xiong $\mathrm{P}$, Zhang $\mathrm{X}$, Wan $\mathrm{H}$, et al. Interface modulation of twodimensional superlattices for efficient overall water splitting. Nano Lett, 2019, 19: 4518-4526

22 Lin S, Dong L, Zhang J, et al. Room-temperature intercalation and $\sim 1000$-fold chemical expansion for scalable preparation of highquality graphene. Chem Mater, 2016, 28: 2138-2146

23 Wang P, Zhang J, Dong L, et al. Interlayer polymerization in chemically expanded graphite for preparation of highly conductive, mechanically strong polymer composites. Chem Mater, 2017, 29: 3412-3422

24 Herrera-Alonso M, Abdala AA, McAllister MJ, et al. Intercalation and stitching of graphite oxide with diaminoalkanes. Langmuir, 2007, 23: 10644-10649

25 Burress JW, Gadipelli S, Ford J, et al. Graphene oxide framework materials: theoretical predictions and experimental results. Angew Chem Int Ed, 2010, 49: 8902-8904

26 Zhang M, Mao Y, Liu G, et al. Molecular bridges stabilize graphene oxide membranes in water. Angew Chem Int Ed, 2020, 59: 16891695

27 Zhang Y, Zhang S, Chung T. Nanometric graphene oxide framework members with enhanced heavy metal removal via nanofiltration. Environ Sci Technol, 2015, 49: 10235-10242

28 Cai J, Chen J, Zeng P, et al. Molecular mechanisms of $\mathrm{CO}_{2}$ adsorption in diamine-cross-linked graphene oxide. Chem Mater, 2019, 31: 3729-3735

29 Zhang X, Ciesielski A, Richard F, et al. Modular graphene-based 3D covalent networks: functional architectures for energy applications. Small, 2016, 12: 1044-1052

30 Yang J, Gong D, Li G, et al. Self-assembly of thiourea-crosslinked graphene oxide framework membranes toward separation of small molecules. Adv Mater, 2018, 30: 1705775

31 Rajesh S, Bose AB. Development of graphene oxide framework membranes via the "from" and "to" cross-linking approach for ion-selective separations. ACS Appl Mater Interfaces, 2019, 11: 27706-27716

32 Yao B, Li C, Ma J, et al. Porphyrin-based graphene oxide frameworks with ultra-large $d$-spacings for the electrocatalyzation of oxygen reduction reaction. Phys Chem Chem Phys, 2015, 17: 19538-19545

33 Zhang J, Liu Q, Ruan Y, et al. Monolithic crystalline swelling of graphite oxide: a bridge to ultralarge graphene oxide with high scalability. Chem Mater, 2018, 30: 1888-1897

34 Diercks CS, Liu Y, Cordova KE, et al. The role of reticular chemistry in the design of $\mathrm{CO}_{2}$ reduction catalysts. Nat Mater, 2018, 17: 301-307

35 Xiao Y, Tian G, Li W, et al. Molecule self-assembly synthesis of porous few-layer carbon nitride for highly efficient photoredox catalysis. J Am Chem Soc, 2019, 141: 2508-2515

36 Hung WS, Tsou CH, De Guzman M, et al. Cross-linking with diamine monomers to prepare composite graphene oxide-framework membranes with varying $d$-spacing. Chem Mater, 2014, 26: 2983-2990 
37 Wu T, Efimenko K, Vlcek P, et al. Formation and properties of anchored polymers with a gradual variation of grafting densities on flat substrates. Macromolecules, 2003, 36: 2448-2453

38 Chen WL, Cordero R, Tran H, et al. 50th anniversary perspective: Polymer brushes: novel surfaces for future materials. Macromolecules, 2017, 50: 4089-4113

39 Zhou X, Chen Z, Yan D, et al. Deposition of Fe-Ni nanoparticles on polyethyleneimine-decorated graphene oxide and application in catalytic dehydrogenation of ammonia borane. J Mater Chem, 2012, 22: 13506-13516

40 Saito Y, Luo X, Zhao C, et al. Filling the gaps between graphene oxide: a general strategy toward nanolayered oxides. Adv Funct Mater, 2015, 25: 5683-5690

41 Chen PY, Liu M, Valentin TM, et al. Hierarchical metal oxide topographies replicated from highly textured graphene oxide by intercalation templating. ACS Nano, 2016, 10: 10869-10879

42 Su Y, Prestat E, Hu C, et al. Self-limiting growth of two-dimensional palladium between graphene oxide layers. Nano Lett, 2019, 19: $4678-4683$

Acknowledgements This work was supported by Shanghai International Collaboration Research Project (19520713900).

Author contributions Ruan $Y$ designed and engineered the samples; Zhao $\mathrm{Z}$ conceived the concept of the manuscript; Ruan $\mathrm{Y}$, Zhao $\mathrm{Z}$ and Li $M$ performed the experiments; Zhao $Z$ wrote the paper with support from Dong L, Zhang J and Lu H. All authors contributed to the general discussion.

Conflict of interest The authors declare no conflict of interest.

Supplementary information Experimental details and supporting data are available in the online version of the paper.

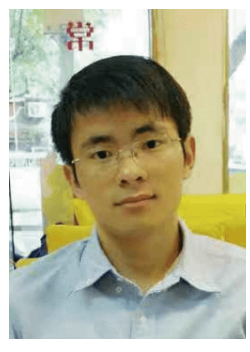

Yingbo Ruan is working at Xi'an Aerospace Composites Research Institute. He worked as a postdoctoral fellow at Fudan University in 20162018. Currently, his research focuses on the synthesis of low-dimensional nanomaterials and their applications in energy storage, catalysis and composite materials.

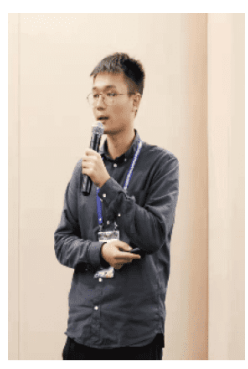

Zedong Zhao is a $\mathrm{PhD}$ candidate at the Department of Macromolecular Science, Fudan University, China. He received his Bachelor degree from Sichuan University in 2017. Currently, his research focuses on the synthesis of low-dimensional nanomaterials and their applications in energy storage and composite materials.

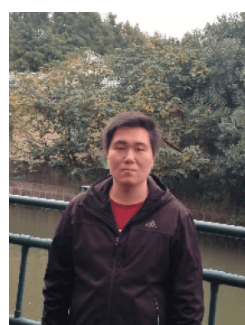

Jiajia Zhang is working at the Department of Macromolecular Science, Fudan University, China. He received his $\mathrm{PhD}$ degree from Fudan University. Currently, his research focuses on the synthesis of low-dimensional nanomaterials and their applications in energy storage and catalysis.

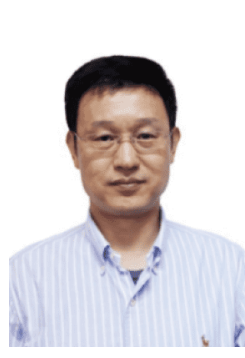

Hongbin Lu is a professor of the Department of Macromolecular Science, Fudan University, China. He received his $\mathrm{PhD}$ degree from Changchun Institute of Applied Chemistry, Chinese Academy of Sciences in 1999. He worked as a postdoctoral fellow at the University of Southern California in America from 2001 to 2004. Currently, his research focuses on the synthesis of low-dimensional nanomaterials and their applications in energy storage, catalysis and composite materials.

具备大层间距、高度周期性类似手风琴的氧化石 墨烯框架用于制备交替排列的二维异质结构

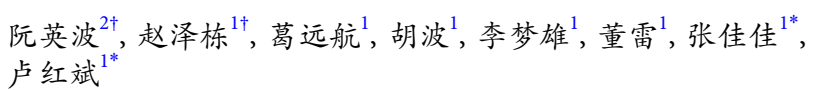

摘要 二维异质结构在集成材料设计中具有广阔的应用前景, 但目 前的合成策略用于多层异质结构构建和大规模生产时仍面临着诸 多挑战. 本论文报导了一种基于非剥离层状氧化石墨烯(LGO)的主 客体策略, 以构建由多层交替排列的石墨烯和金属氧化物纳米片 组成的石墨烯基异质结构. 二维排列的氧化石墨烯和开放的层内 空间使LGO成为构建周期性二维宿主框架的理想平台. 聚醚胺低 聚物被用来共价连接相邻的氧化石墨烯. 伸长的分子链构象使制 得的手风琴石墨烯框架具有良好的结构稳定性、周期性和超大的 层间空间. 开放、排列良好的二维通道与客体材料金属离子前驱 体的高度亲和性, 可限域合成各种高质量的二维石墨烯基异质结 构. 此外, 进一步通过去除石墨烯骨架, 还可以制备各种剥离的超 薄氧化物纳米片. 本工作中提出的灵活可调的层间化学, 为合成一 系列高质量石墨烯-无机/有机二维异质结构提供了新思路. 